\title{
Synthesis of 2-oxopurine adducts with structural resemblance to Efavirenz and DPC 961 as potential NNRT-inhibitors
}

\author{
Geir Andresen, Lise-Lotte Gundersen, and Frode Rise \\ Department of Chemistry, University of Oslo, P.O.Box 1033 Blindern, N-0315 Oslo, Norway \\ E-mail: l.l.gundersen@kjemi.uio.no
}

Dedicated to Professor Kjell Undheim on the Occasion of his $70^{\text {th. }}$ birthday

(received 28 Jun 01; accepted 22 Oct 01; published on the web 30 Oct 01)

\begin{abstract}
6-Cyclopropylethynyl-6-trifluoromethyl dihydro-2-oxopurines have been prepared with completely regioselective addition of trimethyl(trifluoromethyl)silane and cyclopropylethynylmagnesium bromide to 1,9-dialkylated 2-oxopurines as key-steps. The target compounds are structurally related to the Non-Nucleoside reverse transcriptase inhibitors Efavirenz and DPC 961.
\end{abstract}

Keywords: 2-Oxopurines, regioselective addition, non-nucleoside reverse transcriptase inhibitors, Efavirenz, DPC 961

\section{Introduction}

Non-nucleoside reverse transcriptase inhibitors (NNRTI) are of increasing importance for the treatment of AIDS (acquired immune deficiency syndrome). ${ }^{1}$ One of the NNRTIs approved as anti-HIV drug by the FDA is Efavirenz (Sustiva ${ }^{\text {TM }}$ ) (Fig. 1). Several benzopyrimidinone adducts ${ }^{2}$ including the second generation Efavirenz analogs DPC 961 and DPC $963^{3}$ (Fig 1) also inhibit reverse transcriptase. DPC 961 exhibits increased potency and favourable plasma serum protein binding compared to Efavirenz. We have previously prepared 6-substituted adducts of 2oxopurines $^{4}$ and as a continuance of this work we now report the synthesis of 6,6-disubstituted 2oxopurine adducts with structural resemblance to Efavirenz, DPC 961 and DPC 963. 


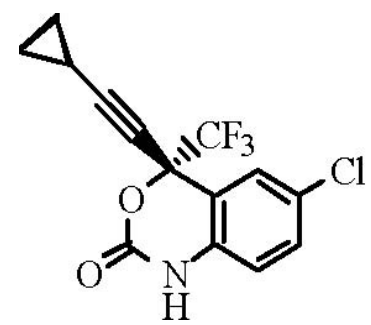

Efavirenz

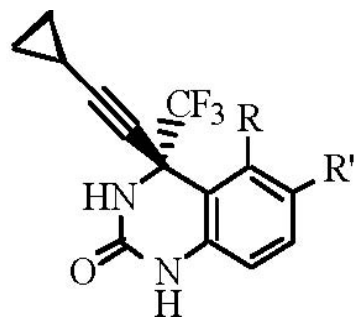

DPC 961: $\mathrm{R}=\mathrm{H}, \mathrm{R}^{\prime}=\mathrm{Cl}$

DPC 963: $\mathrm{R}=\mathrm{R}^{\prime}=\mathrm{F}$

Figure 1

\section{Results and Discussion}

The synthesis of the target 2-oxopurines is outlined in Scheme 1. The 1,9-dialkylated 2oxopurines 1 were prepared as previously reported ${ }^{4,5}$ and reacted with trimethyl(trifluoromethyl)silane $\left(\mathrm{TMSCF}_{3}\right)$. Reactions of $\mathrm{TMSCF}_{3}$ with carbonyl compounds are often performed in the presence of tetrabutylammonium fluoride (TBAF), ${ }^{6}$ but attempts to employ a fluoride source (TBAF, $\mathrm{Me}_{4} \mathrm{NF}$ or $\mathrm{KF}$ ) in the addition to the purine 1a met with little success. Furthermore, fluoride ions would have been incompatible with the silicon based $N$ protecting groups in compound $1 \mathrm{~b}$. Potassium carbonate is known to catalyze the reaction between trifluoromethylsilanes and quinones, ${ }^{7}$ and in the presence of $\mathrm{K}_{2} \mathrm{CO}_{3}$, the silane reacted with the 2-oxopurines in the 6-position exclusively to give compounds $\mathbf{2 a}$ and $\mathbf{2} \mathbf{b}$ in 58 and $62 \%$ yields respectively. The regiochemistry of compounds 2 as well as compound $\mathbf{4}$ below were established by HMQC and HMBC NMR spectroscopy. ${ }^{4}$ After purification, adducts $\mathbf{2}$ were easily rearomatized with $\mathrm{MnO} 2$ to give compounds 6 . The use of DDQ in these oxidations resulted in complex mixtures and oxidation of crude 2 with $\mathrm{MnO}_{2}$ was also less successful. The alkynyl substituent in compounds $\mathbf{4 a}$ and $\mathbf{4 b}$ were introduced when the purines $\mathbf{3 a}$ or $\mathbf{3 b}$ were reacted with cyclopropylethynylmagnesium bromide (generated in situ from cyclopropylethyne ${ }^{8}$ and ethylmagnesium bromide). Again complete regioselectivity in the addition was observed.

Even though both addition reactions took place in the purine 6-position, a carbon substituent can also easily be introduced in the 8-position as demonstrated in the synthesis of the 8phenylpurine 4c. Bromination of the trifluoro compound $\mathbf{3 b}$ gave the 8bromopurine 3c which easily reacted with phenylboronic acid in a Suzuki type coupling. ${ }^{9,10}$ The 8 -substituted 2oxopurine 3d did not add cyclopropylethynylmagnesium bromide as described for compound 3a and $\mathbf{3 b}$ above, but the reaction with the corresponding organolithium reagent gave the desired adduct $4 \mathbf{c}$ in $45 \%$ yields. No regioisomers were detected in the reaction mixture. Finally the silicon based protecting groups in compounds $\mathbf{4 b}$ and $\mathbf{4 c}$ were removed with $\mathrm{Me}_{4} \mathrm{NF}$ to give the DPC 961 analogs 5.

Reverse introduction of the purine 6-substituents was also attempted. Compounds 7 were readily available from addition of the ethynylmagnesium bromide reagent followed by oxidation, 
but reaction of the alkynylpurines 7 with $\mathrm{TMSCF}_{3}$ resulted only in complex mixtures.

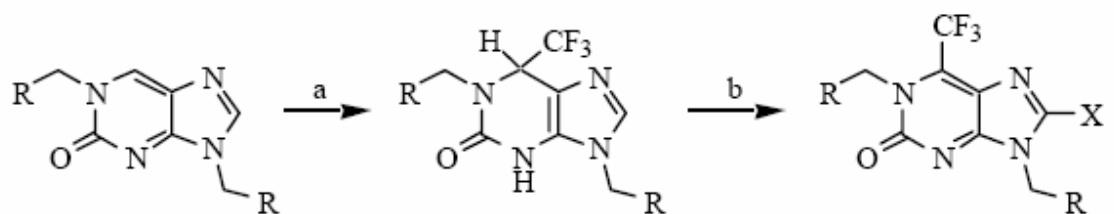

$$
\begin{array}{lll}
\text { la: }: R=P h & \text { 2a: }:=P h, 58 \% & \text { 3a: }: R=P h, X=H, 84 \% \\
\text { lb: }:=\text { OTBDMS } & \text { 2b: }: \text { = OTBDMS, } 62 \% & \text { 3b: } R=\text { OTBDMS, } X=H, 71 \%
\end{array}
$$

lb: $\mathrm{R}=$ OTBDMS $\quad 2 \mathrm{~b}: \mathrm{R}=$ OTBDMS, $62 \%$$$
\text { 3c: } R=\text { OTBDMS, } X=B r, 86 \%
$$

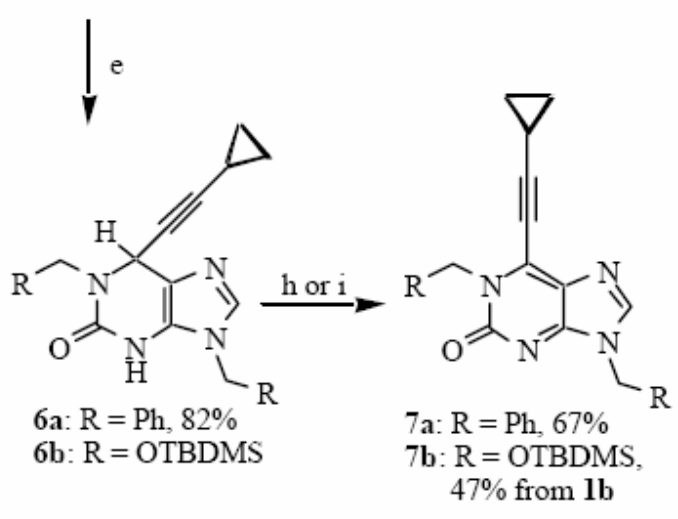

3d: $\mathrm{R}=$ OTBDMS, $\mathrm{X}=\mathrm{Ph}, 81 \% \longleftarrow \mathrm{d}$

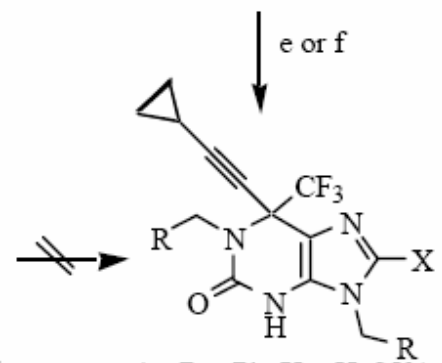

4a: $\mathrm{R}=\mathrm{Ph}, \mathrm{X}=\mathrm{H}, 85 \%$

4b: $\mathrm{R}=$ OTBDMS, $\mathrm{X}=\mathrm{H}, 88 \%$

4c: $\mathrm{R}=$ OTBDMS, $\mathrm{X}=\mathrm{Ph}, 45 \%$

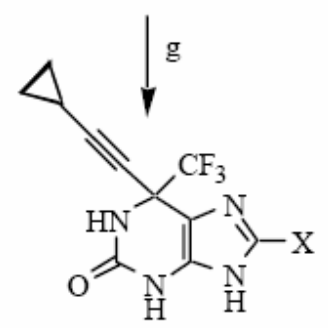

5a, $\mathrm{X}=\mathrm{H}, 69 \%$

5b, $\mathrm{X}=\mathrm{Ph}, 70 \%$

Scheme 1. (a) $\mathrm{TMSCF}_{3}, \mathrm{~K}_{2} \mathrm{CO}_{3}, \mathrm{DMF}, 0{ }^{\circ} \mathrm{C}$; (b) $\mathrm{MnO}_{2}, \mathrm{PhH}$; (c) $\mathrm{Br}_{2}, \mathrm{AcONa}, \mathrm{AcOH}$; (d) $\mathrm{PhB}(\mathrm{OH})_{2}, \mathrm{Pd}\left(\mathrm{Ph}_{3} \mathrm{P}\right)_{4}, \mathrm{~K}_{2} \mathrm{CO}_{3}, \mathrm{PhMe}, 90{ }^{\circ} \mathrm{C}$; (e) cyclopropylCCMgBr, THF, $-78{ }^{\circ} \mathrm{C}$; (f) cyclopropylCCLi, THF, $-78{ }^{\circ} \mathrm{C}$; (g) $\mathrm{Me}_{4} \mathrm{NF}, \mathrm{MeCN}$; (h) $\mathrm{MnO}_{2} \mathrm{CH}_{2} \mathrm{Cl}_{2}$; (i) DDQ, $\mathrm{PhH}$.

\section{Experimental Section}

General Procedures. The ${ }^{1} \mathrm{H}-\mathrm{NMR}$ spectra were recorded at $500 \mathrm{MHz}$ with a Bruker Avance DRX 500 instrument, at $300 \mathrm{MHz}$ with a Bruker Avance DPX 300 instrument or at $200 \mathrm{MHz}$ with a Bruker Avance DPX 200 instrument. The ${ }^{1} \mathrm{H}$ decoupled ${ }^{13} \mathrm{C}$-NMR spectra were recorded at 125,75 or $50 \mathrm{MHz}$ using the above mentioned spectrometers. ${ }^{19} \mathrm{~F}-\mathrm{NMR}$ spectra were recorded at $188 \mathrm{MHz}$ at the Bruker Avance DPX 200 instrument. $J$ values are given in $\mathrm{Hz} .{ }^{1} \mathrm{H}-$ and ${ }^{13} \mathrm{C}$ NMR spectra were referenced to the solvent and ${ }^{19} \mathrm{~F}-\mathrm{NMR}$ spectra were referenced to fluorotrichloromethane (freon 11). Mass spectra under electron impact conditions (EI) were 
recorded at $70 \mathrm{eV}$ ionising voltage with a VG Prospec instrument, and are presented as $\mathrm{m} / \mathrm{z}(\%$ rel. int.). Methane was used for chemical ionisation (CI). Elemental analyses were performed by Ilse Beetz Mikroanalytisches Laboratorium, Kronach, Germany. Melting points are uncorrected. Silica gel for flash chromatography was purchased from Merck, Darmstadt, Germany (Merck No. 9385). Analytical thin layer chromatography was performed with E. Merck silica gel $60 \mathrm{~F}_{254}$ $0.25 \mathrm{~mm}$ plates (Merck No. 1.05554).

Materials. THF was distilled from sodium-benzophenone, and DMF from $\mathrm{BaO}$. Benzene and toluene were dried over a sodium-wire. $\mathrm{CH}_{2} \mathrm{Cl}_{2}$ was predried with $\mathrm{CaCl}_{2}$, distilled from $\mathrm{CaH}_{2}$ and stored over $4 \AA$ molsieve. $\mathrm{MeCN}$ was distilled from $\mathrm{CaH}_{2}$ and stored over $4 \AA$ molsieve. Tetramethylammonium fluoride tetrahydrate was dried by azeotropic distillation with abs. EtOH and the residue was dissolved in dry MeCN. 1,9-dibenzyl-1,9-dihydro- $2 H$-purin-2-one $1 \mathrm{a},{ }^{4}{ }_{1,9-}$ dihydro-1,9-di[(tert-butyldimethylsilyloxy)methyl]-2H-purin-2-one $1 \mathrm{~b}^{5}$ and cyclopropylethyne ${ }^{8}$ were prepared according to literature procedures.

All other reagents were commercially available and used as received. Solvent mixtures are defined as volume ratios (v/v).

1,9-Dibenzyl-1,3,6,9-tetrahydro-6-trifluoromethyl-2H-purin-2-one (2a). To a mixture of 1,9dibenzyl-1,9-dihydro-2 $\mathrm{H}$-purin-2-one (479 mg, $1.5 \mathrm{mmol}$ ) and $\mathrm{K}_{2} \mathrm{CO}_{3}$ (1.05 g, $7.5 \mathrm{mmol}$ ) in dry DMF $(50 \mathrm{~mL})$ under $\mathrm{N}_{2}$ at $0{ }^{\circ} \mathrm{C}$ was added dropwise $\mathrm{TMSCF}_{3}(3.8 \mathrm{~mL}, 7.5 \mathrm{mmol}, 2 \mathrm{M}$ solution in THF). The mixture was stirred for $17 \mathrm{~h}$ while slowly reaching ambient temperature and diluted with saturated aqueous ammonium chloride $(50 \mathrm{~mL})$ and EtOAc $(100 \mathrm{~mL})$. The phases were separated and the aqueous phase extracted with EtOAc $(2 \times 100 \mathrm{~mL})$. The combined organic phases were washed with saturated aqueous sodium chloride $(50 \mathrm{~mL})$, dried $\left(\mathrm{MgSO}_{4}\right)$ and evaporated in vacuo. The product was isolated by flash chromatography on silica gel eluting with EtOAc/hexane (1:1) to give the title compound (340 mg, 58\%) as a colorless oil; ${ }^{1} \mathrm{H}-\mathrm{NMR}(200$ $\left.\mathrm{MHz} ; \mathrm{CDCl}_{3}\right) \delta 10.28(1 \mathrm{H}, \mathrm{s}, \mathrm{NH}), 7.3-7.1(10 \mathrm{H}, \mathrm{m}, \mathrm{Ph}), 7.11(1 \mathrm{H}, \mathrm{s}, 8-\mathrm{H}), 5.45$ [1H, d, J 15.4, $\mathrm{HB}$ in $\left.\mathrm{N}(1) \mathrm{CH}_{2}\right], 5.03\left[2 \mathrm{H}, \mathrm{s}, \mathrm{N}(9) \mathrm{CH}_{2}\right], 4.91(1 \mathrm{H}, \mathrm{q}, J 5.8,6-\mathrm{H}), 4.16\left[1 \mathrm{H}, \mathrm{d}, J 15.4, \mathrm{H}_{\mathrm{A}}\right.$ in $\left.\mathrm{N}(1) \mathrm{CH}_{2}\right] ;{ }^{19} \mathrm{FNMR}\left(188 \mathrm{MHz} ; \mathrm{CDCl}_{3}\right) \delta-75.41\left(\mathrm{~d}, J 5.7, \mathrm{CF}_{3}\right) ;{ }^{13} \mathrm{C}-\mathrm{NMR}\left(75 \mathrm{MHz} ; \mathrm{CDCl}_{3}\right) \delta$ 154.7 (2-C), 135.6 (C in Ph), 134.9 (C in Ph), 133.0 (8-C), 129.6 (4-C), 129.0 (CH in Ph), 128.8 $(\mathrm{CH}$ in $\mathrm{Ph}), 128.8(\mathrm{CH}$ in $\mathrm{Ph}), 128.3(\mathrm{CH}$ in $\mathrm{Ph}), 127.9(\mathrm{CH}$ in $\mathrm{Ph}), 127.7(\mathrm{CH}$ in $\mathrm{Ph}), 127.2(\mathrm{CH}$ in $\mathrm{Ph}$ ), 126.4 (q, $J$ 285.3, $\mathrm{CF}_{3}$ ), 108.5 (5-C), 57.0 (q, $J$ 31.9, 6-C), 50.3 [N(1)CH $\left.\mathrm{CH}_{2}\right], 47.7$ [N(9) $\mathrm{CH}_{2}$ ]; MS (EI) m/z: $386\left(\mathrm{M}^{+}, 11 \%\right), 318$ (13), 317 (59), 150 (6), 132 (6), 104 (6), 99 (15), 92 (9), 91 (100), 65 (11); Anal. Calcd for $\mathrm{C}_{20} \mathrm{H}_{17} \mathrm{~F}_{3} \mathrm{~N}_{4} \mathrm{O}$ : C, 62.17; H, 4.44. Found: C, 62.25; H, 4.50 .

\section{1,9-Di[(tert-butyldimethylsilyloxy)methyl]-1,3,6,9-tetrahydro-6-trifluoromethyl-2H-purin-}

2-one (2b). To a mixture of 1,9-dihydro-1,9-di[(tert-butyldimethylsilyloxy)methyl]-2H-purin-2one (348 mg, $0.82 \mathrm{mmol})$ and $\mathrm{K}_{2} \mathrm{CO}_{3}(565 \mathrm{mg}, 4.1 \mathrm{mmol})$ in dry DMF $(35 \mathrm{~mL})$ under $\mathrm{N}_{2}$ at $0{ }^{\circ} \mathrm{C}$ was added dropwise $\mathrm{TMSCF}_{3}(2.05 \mathrm{~mL}, 4.1 \mathrm{mmol}, 2 \mathrm{M}$ solution in THF). The mixture was stirred for $17 \mathrm{~h}$ while slowly reaching ambient temperature and diluted with saturated aqueous ammonium chloride $(30 \mathrm{~mL})$ and EtOAc $(50 \mathrm{~mL})$. The phases were separated and the aqueous 
phase extracted with EtOAc $(2 \times 50 \mathrm{~mL})$. The combined organic phases were washed with saturated sodium chloride $(30 \mathrm{~mL})$, dried $\left(\mathrm{MgSO}_{4}\right.$ and evaporated in vacuo. The product was isolated by flash chromatography on silica gel eluting with EtOAc/hexane (1:2) to give the title compound (250 mg, 62\%) as colorless crystals; $170-3{ }^{\circ} \mathrm{C} ;{ }^{\prime} \mathrm{H}-\mathrm{NMR}\left(300 \mathrm{MHz} ; \mathrm{CDCl}_{3} \delta 8.97\right.$ $(1 \mathrm{H}, \mathrm{s}, \mathrm{NH}), 7.25(1 \mathrm{H}, \mathrm{s}, 8-\mathrm{H}), 5.69\left[1 \mathrm{H}, \mathrm{d}, J\right.$ 9.3, $\mathrm{H}_{\mathrm{B}}$ in $\mathrm{N}(1) \mathrm{CH}_{2} 5.42\left[2 \mathrm{H}, \mathrm{s}, \mathrm{N}(9) \mathrm{CH}_{2}, 5.31\right.$ $(1 \mathrm{H}, \mathrm{q}, J 5.9,6 \mathrm{H}), 4.76\left[1 \mathrm{H}, \mathrm{d}, J 9.3, \mathrm{H}_{\mathrm{A}}\right.$ in $\mathrm{N}(1) \mathrm{CH}_{2}, 0.85(9 \mathrm{H}, \mathrm{s}, \mathrm{Me}$ in $t-\mathrm{Bu}), 0.84(9 \mathrm{H}, \mathrm{s}, \mathrm{Me}$ in t-Bu), 0.08 (3H, s, SiMe), 0.06 (3H, s, SiMe), 0.04 (3 H, s, SiMe), 0.003 (3H, s, SiMe); ${ }^{19} \mathrm{~F}-\mathrm{NMR}$ $\left(188 \mathrm{MHz} ; \mathrm{CDCl}_{3}\right) \delta-75.52\left(\mathrm{~d}, J 6.6, \mathrm{CF}_{3}\right) ;{ }^{13} \mathrm{C}-\mathrm{NMR}\left(75 \mathrm{MHz} ; \mathrm{CDCl}_{3}\right) \delta 153.6(2-\mathrm{C}), 132.3$ (8C), 128.8 (4-C), 124.4 (q, J 284.3, $\left.\mathrm{CF}_{3}\right), 109.4$ (5-C), 70.7 [N(1)CH $2,68.4$ [N(9) $\left.\mathrm{CH}_{2}\right], 54.4$ (q, $J$ $32.4,6-\mathrm{C}), 25.6(\mathrm{Me}$ in $t-\mathrm{Bu}), 25.3(\mathrm{Me}$ in $t-\mathrm{Bu}), 18.0(\mathrm{C}$ in $t-\mathrm{Bu}), 17.8(\mathrm{C}$ in $t-\mathrm{Bu}),-5.2(\mathrm{SiMe})$, -5.5 (SiMe); MS (EI) m/z: 494 (M+, 3\%), 438 (29), 437 (100), 305 (42), 275 (37), 193 (18), 165 (31), 89 (22), 73 (41); Anal. Calcd for $\mathrm{C}_{20} \mathrm{H}_{37} \mathrm{~F}_{3} \mathrm{~N}_{43} \mathrm{Si}_{2} \mathrm{C}, 48.56$; H, 7.54. Found: C, 48.55; H, 7.44 .

1,9-Dibenzyl-1,9-dihydro-6-trifluoromethyl-2H-purin-2-one (3a). To a solution of 1,9dibenzyl-1,3,6,9-tetrahydro-6-trifluoromethyl-2H-purin-2-one $(120 \mathrm{mg}, 0.31 \mathrm{mmol})$ in dry benzene $(30 \mathrm{~mL})$ was added $\mathrm{MnO}_{2}(1.2 \mathrm{~g}, 13.8 \mathrm{mmol})$. The resulting mixture was stirred at ambient temperature for 23 hours, filtered and the product was isolated by flash chromatography on silica gel eluting with EtOAc/hexane (1:1) followed by EtOAc/hexane (3:2) to give the title compound (100 mg, 84\%) as colorless crystals; $122-4{ }^{\circ} \mathrm{C} ;{ }^{1} \mathrm{H}-\mathrm{NMR}\left(200 \mathrm{MHz} ; \mathrm{CDCl}_{3}\right) \delta 7.95$ $(1 \mathrm{H}, \mathrm{s}, 8-\mathrm{H}), 7.4-7.1(10 \mathrm{H}, \mathrm{m}, \mathrm{Ph}), 5.51\left[2 \mathrm{H}, \mathrm{s}, \mathrm{N}(1) \mathrm{CH}_{2}\right], 5.24$ [2H, s, N(9) $\left.\mathrm{CH}_{2}\right] ;{ }^{19} \mathrm{~F}-\mathrm{NMR}(188$ $\left.\mathrm{MHz} ; \mathrm{CDCl}_{3}\right) \delta-59.45\left(\mathrm{~s}, \mathrm{CF}_{3}\right) ;{ }^{13} \mathrm{CNMR}\left(75 \mathrm{MHz} ; \mathrm{CDCl}_{3}\right) \delta 160.6$ (4-C), 155.1 (2-C), 149.9 (q, J 2.1, 8-C) , 135.9 (q, J 36.1, 6-C), 135.4 (C in Ph), 134.2 (C in Ph), 129.2 (CH in Ph), 128.8 $(\mathrm{CH}$ in $\mathrm{Ph}), 128.5(\mathrm{CH}$ in $\mathrm{Ph}), 128.3(\mathrm{CH}$ in $\mathrm{Ph}), 127.5(\mathrm{CH}$ in $\mathrm{Ph}), 126.2(\mathrm{CH}$ in $\mathrm{Ph}), 122.8$ (5-

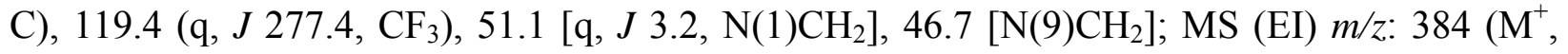
71\%), 294 (16), 293 (84), 224 (21), 91 (100), 65 (12); Anal. Calcd for $\mathrm{C}_{20} \mathrm{H}_{15} \mathrm{~F}_{3} \mathrm{~N} 4 \mathrm{O}$ : C, 62.50; H, 3.93. Found: C, 62.28; H, 3.88.

\section{1,9-Dihydro-1,9-di[(tert-butyldimethylsilyloxy)methyl]-6-trifluoromethyl-2H-purin-2-one}

(3b). To a solution of 1,9-di[(tert-butyldimethylsilyloxy)methyl]-1,3,6,9-tetrahydro-6trifluoromethyl-2H-purin-2-one $(105 \mathrm{mg}, 0.21 \mathrm{mmol})$ in dry benzene $(25 \mathrm{~mL})$ was added $\mathrm{MnO}_{2}$ $(1.0 \mathrm{~g}, 11.5 \mathrm{mmol})$. The resulting mixture was stirred at ambient temperature for 24 hours, filtered and the product was isolated by flash chromatography on silica gel eluting with EtOAc/hexane (1:2) to give the title compound (74 mg, 71\%) as colorless crystals; $99-102{ }^{\circ} \mathrm{C}$; ${ }^{1} \mathrm{H}-\mathrm{NMR}\left(200 \mathrm{MHz} ; \mathrm{CDCl}_{3}\right) \delta 8.06(1 \mathrm{H}, \mathrm{s}, 8-\mathrm{H}), 5.74$ [2H, s, N(1)CH $\mathrm{CH}_{2}, 5.54$ [2H, s, N(9) $\mathrm{CH}_{2}$ ], $0.87(9 \mathrm{H}, \mathrm{s}, \mathrm{Me}$ in $t-\mathrm{Bu}), 0.86(9 \mathrm{H}, \mathrm{s}, \mathrm{Me}$ in $t-\mathrm{Bu}), 0.15(6 \mathrm{H}, \mathrm{s}, 2 \times \mathrm{SiMe}), 0.12(6 \mathrm{H}, \mathrm{s}, 2 \times \mathrm{SiMe})$; ${ }^{19} \mathrm{~F}-\mathrm{NMR}\left(188 \mathrm{MHz} ; \mathrm{CDCl}_{3}\right) \delta-58.95\left(\mathrm{~s}, \mathrm{CF}_{3}\right) ;{ }^{13} \mathrm{C}-\mathrm{NMR}\left(75 \mathrm{MHz} ; \mathrm{CDCl}_{3}\right) \delta 160.4(4-\mathrm{C})$, 154.6 (2-C), 149.7 (8-C), 135.6 (q, J 36.8, 6C), 125.5 (q, J 400.4, $\mathrm{CF}_{3}$ ), 121.3 (5-C), 71.1 [q, $J$ $\left.3.3, \mathrm{~N}(1) \mathrm{CH}_{2}\right], 66.9\left[\mathrm{~N}(9) \mathrm{CH}_{2}\right], 25.4(\mathrm{Me}$ in $t-\mathrm{Bu}), 18.0(\mathrm{C}$ in $t-\mathrm{Bu}), 17.9(\mathrm{C}$ in $t-\mathrm{Bu}),-5.3$ (SiMe), -5.5 (SiMe); MS (EI) m/z: 492 (M+, 0.3\%), 435 (22), 407 (11), 406 (28), 405 (100), 99 (12), 89 (13), 73 (22); Anal. Calcd for $\mathrm{C}_{20} \mathrm{H}_{35} \mathrm{~F}_{3} \mathrm{~N}_{4} \mathrm{O}_{3} \mathrm{Si}_{2}$ : C, 48.75; H, 7.16. Found: C, 48.47; H, 7.54 . 


\section{8-Bromo-1,9-dihydro-1,9-di[(tert-butyldimethylsilyloxy)methyl]-6-trifluoromethyl-2H-}

purin-2-one (3c). To a solution of 1,9-dihydro-1,9-di[(tert-butyldimethylsilyloxy)methyl]-6trifluoromethyl-2H-purin-2-one $(216 \mathrm{mg}, 0.44 \mathrm{mmol})$ in acetic acid $(12 \mathrm{~mL})$ and sodium acetate $(1.2 \mathrm{~g})$ was added $\mathrm{Br}_{2}(0.050 \mathrm{~mL}, 0.97 \mathrm{mmol})$. The solution was stirred for 2 hours at room temperature before diluting with EtOAc $(40 \mathrm{~mL})$ and water $(40 \mathrm{~mL})$. The resulting mixture was stirred vigorously and aqueous $\mathrm{NaHSO}_{3}(5 \%)$ was added until the solution became colorless. The phases were separated and the aqueous phase extracted with EtOAc $(2 \times 40 \mathrm{~mL})$. The combined organic phases were washed with saturated aqueous sodium chloride $(30 \mathrm{~mL})$, dried $\left(\mathrm{MgSO}_{4}\right)$ and evaporated in vacuo. The product was isolated by flash chromatography on silica gel eluting with EtOAc/hexane (1:6) to give the title compound (215 $\mathrm{mg}, 86 \%)$ as colorless crystals; mp 99-103 ${ }^{\circ} \mathrm{C}$; ${ }^{1} \mathrm{H}-\mathrm{NMR}\left(300 \mathrm{MHz} ; \mathrm{CDCl}_{3}\right) \delta 5.71\left[2 \mathrm{H}, \mathrm{s}, \mathrm{N}(1) \mathrm{CH}_{2}\right] 5.54[2 \mathrm{H}, \mathrm{s}$, $\left.\mathrm{N}(9) \mathrm{CH}_{2}\right], 0.86(9 \mathrm{H}, \mathrm{s}, \mathrm{Me}$ in $t-\mathrm{Bu}), 0.85(9 \mathrm{H}, \mathrm{s}, \mathrm{Me}$ in $t-\mathrm{Bu}), 0.15(6 \mathrm{H}, \mathrm{s}, 2 \times \mathrm{SiMe}), 0.14(6 \mathrm{H}, \mathrm{s}$, $2 \times \mathrm{SiMe}) ;{ }^{19} \mathrm{~F}-\mathrm{NMR}\left(188 \mathrm{MHz} ; \mathrm{CDCl}_{3}\right) \delta-58.97\left(\mathrm{~s}, \mathrm{CF}_{3}\right) ;{ }^{13} \mathrm{C}-\mathrm{NMR}\left(75 \mathrm{MHz} ; \mathrm{CDCl}_{3}\right) \delta 161.0$ (4-C), 154.3 (2-C), 139.3 (8-C), 134.3 (q, J 36.9, 6-C), 122.9 (5-C), 119.3 (q, J 277.5, $\mathrm{CF}_{3}$ ), 71.2 $\left[\mathrm{N}(1) \mathrm{CH}_{2}\right], 66.6\left[\mathrm{~N}(9) \mathrm{CH}_{2}\right], 25.5(\mathrm{Me}$ in $t-\mathrm{Bu}), 18.1(\mathrm{C}$ in $t-\mathrm{Bu}), 18.0(\mathrm{C}$ in $t-\mathrm{Bu}),-5.2(\mathrm{SiMe}),-$ 5.4 (SiMe); MS (CI) m/z: 573 (M+1, 100\%), $572\left(\mathrm{M}^{+}, 29\right), 557$ (29), 543 (27), 515 (30), 429 (27), 145 (39), 89 (26), 79 (36); Anal. Calcd for $\mathrm{C}_{20} \mathrm{H}_{34} \mathrm{BrF}_{3} \mathrm{~N}_{4} \mathrm{O}_{3} \mathrm{Si} 2$ : C,42.02; H, 6.00. Found: C, 42.13; H, 6.13 .

\section{1,9-Dihydro-1,9-di[(tert-butyldimethylsilyloxy)methyl]-8-phenyl-6-trifluoromethyl-2H-}

purin-2-one (3d). A mixture of 8-bromo-1,9-dihydro-1,9-di[(tert-
butyldimethylsilyloxy)methyl]-6-trifluoromethyl-2H-purin-2-one $(190 \mathrm{mg}, \quad 0.33 \mathrm{mmol})$, phenylboronic acid (61 mg, $0.50 \mathrm{mmol}$ ), anhydrous $\mathrm{K}_{2} \mathrm{CO}_{3}(71 \mathrm{mg}, 0.51 \mathrm{mmol}$ ), tetrakis(triphenylphosphine)palladium(0) $(20 \mathrm{mg}, 0.017 \mathrm{mmol})$ and dry toluene $(6 \mathrm{~mL})$ was stirred under $\mathrm{N}_{2}$ at $90{ }^{\circ} \mathrm{C}$ for $16 \mathrm{~h}$, evaporated and chromatographed on silica gel eluting with EtOAc/hexane (1:6) to give the title compound (153 mg, 81\%) as colorless crystals; $\mathrm{mp} 204-5{ }^{\circ} \mathrm{C}$ $\left(\mathrm{CH}_{2} \mathrm{Cl}_{2}\right.$ /hexane); $\mathrm{R}_{\mathrm{F}} 0.24$ (1:4 EtOAc/hexane); ${ }^{1} \mathrm{H}-\mathrm{NMR}\left(300 \mathrm{MHz} ; \mathrm{CDCl}_{3}\right) \delta 8.1(2 \mathrm{H}, \mathrm{m}, \mathrm{CH}$ in $\mathrm{Ph})$, 7.6-7.5 (3H, m, CH in $\mathrm{Ph}), 5.75$ [2H, s, N(9) $\left.\mathrm{CH}_{2}\right], 5.57$ [2H, s, N(1) $\left.\mathrm{CH}_{2}\right], 0.88(9 \mathrm{H}, \mathrm{s}, \mathrm{Me}$ in $t$-Bu), 0.87 (9H, s, Me in $t$-Bu), $0.17(12 \mathrm{H}, \mathrm{s}, 4 \times \mathrm{SiMe}) ;{ }^{19} \mathrm{~F}-\mathrm{NMR}(188 \mathrm{MHz}$; CDCl3) $\delta-58.56$ $\left(\mathrm{s}, \mathrm{CF}_{3}\right) ;{ }^{13} \mathrm{C}-\mathrm{NMR}\left(75 \mathrm{MHz} ; \mathrm{CDCl}_{3}\right.$ ) $\delta 162.7$ (4-C), 161.0 (8-C), 154.8 (2-C), 134.0 (q, J 36.5, 6C), $131.9(\mathrm{C}$ in $\mathrm{Ph}), 129.4$ ( $\mathrm{CH}$ in $\mathrm{Ph}), 128.9$ (CH in $\mathrm{Ph}), 128.0$ (C in $\mathrm{Ph}), 123.1$ (5-C), 119.7 (q, $J$ 277.4, $\left.\mathrm{CF}_{3}\right), 71.0\left[\mathrm{~N}(1) \mathrm{CH}_{2}\right], 65.8\left[\mathrm{~N}(9) \mathrm{CH}_{2}\right], 25.5(\mathrm{Me}$ in $t-\mathrm{Bu}), 18.1(\mathrm{C}$ in $t-\mathrm{Bu}), 18.0(\mathrm{C}$ in $t-$ $\mathrm{Bu}$ ), -5.1 (SiMe), -5.4 (SiMe); MS (CI) m/z: 569 (M+1, 7\%), 511 (100), 481 (61), 262 (4). HRMS: Found $511.1801\left(\mathrm{M}^{+}-t-\mathrm{Bu}\right)$, calc. for $\mathrm{C}_{22} \mathrm{H}_{30} \mathrm{~F}_{3} \mathrm{~N}_{4} \mathrm{O} 3 \mathrm{Si}_{2} 511.1809$.

\section{1,9-Dibenzyl-6-cyclopropylethynyl-1,3,6,9-tetrahydro-6-trifluoromethyl-2H-purin-2-one}

(4a). To a solution of 1,9-dibenzyl-1,9-dihydro-6-trifluoromethyl-2 $\mathrm{H}$-purin-2-one (90 mg, 0.23 mmol) in dry THF ( $4 \mathrm{~mL}$ ) under $\mathrm{N}_{2}$ at $-78{ }^{\circ} \mathrm{C}$ was added cyclopropylethynylmagnesium bromide [generated in situ by adding cyclopropylethyne (140 mg, $1.27 \mathrm{mmol}, 60 \%$ solution in cyclohexane $)$ in dry THF ( $1 \mathrm{~mL})$ to a solution of ethylmagnesium bromide $(1.1 \mathrm{~mL}, 1.15 \mathrm{mmol}$, $1.0 \mathrm{M}$ solution in THF) in dry THF $(2 \mathrm{~mL})$ under $\mathrm{N}_{2}$ and refluxing the resulting mixture for 2 hours before cooling to ambient temperature]. After stirring for 30 minutes at $-78{ }^{\circ} \mathrm{C}$ and 30 
minutes at ambient temperature, saturated aqueous ammonium chloride $(10 \mathrm{~mL})$ was added to the reaction mixture and the phases were separated. The aqueous phase was extracted with $\mathrm{CH}_{2} \mathrm{Cl}_{2}(3 \times 10 \mathrm{~mL})$ and the combined organic phases were dried $\left(\mathrm{MgSO}_{4}\right)$ and evaporated in vacuo. Flash chromatography on silica gel eluting with EtOAc/hexane (1:1) gave the title compound (89 mg, 85\%) as colorless crystals; $212-3{ }^{\circ} \mathrm{C} ;{ }^{1} \mathrm{H}-\mathrm{NMR}\left(500 \mathrm{MHz} ; \mathrm{CDCl}_{3}\right) \delta 10.64$ $(1 \mathrm{H}, \mathrm{s}, \mathrm{NH}), 7.3-7.0(11 \mathrm{H}, \mathrm{m}, \mathrm{CH}$ in $\mathrm{Ph}$ and $8-\mathrm{H}), 4.82\left[1 \mathrm{H}, \mathrm{d}, J 16.1, \mathrm{HB}\right.$ in $\left.\mathrm{N}(1) \mathrm{CH}_{2}\right], 4.78$ $\left[1 \mathrm{H}, \mathrm{d}, J 16.1, \mathrm{H}_{\mathrm{A}}\right.$ in $\left.\mathrm{N}(1) \mathrm{CH}_{2}\right], 4.68\left[1 \mathrm{H}, \mathrm{d}, J 15.4, \mathrm{H}_{\mathrm{B}}\right.$ in $\left.\mathrm{N}(9) \mathrm{CH}_{2}\right], 4.64\left[1 \mathrm{H}, \mathrm{d}, J 15.4, \mathrm{H}_{\mathrm{A}}\right.$ in $\left.\mathrm{N}(9) \mathrm{CH}_{2}\right], 1.20\left(1 \mathrm{H}, \mathrm{m}, \mathrm{CH}\right.$ in cyclopropyl), $0.63\left(2 \mathrm{H}, \mathrm{m}, \mathrm{CH}_{2}\right.$ in cyclopropyl), $0.43(2 \mathrm{H}, \mathrm{m}$, $\mathrm{CH}_{2}$ in cyclopropyl), ${ }^{19} \mathrm{~F}-\mathrm{NMR}\left(188 \mathrm{MHz} ; \mathrm{CDCl}_{3}\right) \delta-79.44\left(\mathrm{~s}, \mathrm{CF}_{3}\right) ;{ }^{13} \mathrm{C}-\mathrm{NMR}(75 \mathrm{MHz}$; $\left.\mathrm{CDCl}_{3}\right) \delta 153.7$ (2-C); $138.5(\mathrm{C}$ in $\mathrm{Ph}), 134.6(\mathrm{C}$ in $\mathrm{Ph}), 132.7$ (8-C), $128.9(\mathrm{CH}$ in $\mathrm{Ph}$ or $4-\mathrm{C})$, 126.1 (CH in $\mathrm{Ph}$ or 4-C), 123.9 (q, $J$ 288.7, $\mathrm{CF}_{3}$ ), 110.3 (5-C), 93.5 (三C-cyclopropyl), 66.2 (Pur$C \equiv), 62.9$ (q, $J 32.3,6-\mathrm{C}), 49.6\left[\mathrm{~N}(1) \mathrm{CH}_{2}\right], 47.6\left[\mathrm{~N}(9) \mathrm{CH}_{2}\right], 8.2\left(\mathrm{CH}_{2}\right.$ in cyclopropyl), $8.1\left(\mathrm{CH}_{2}\right.$ in cyclopropyl), -0.6 (CH in cyclopropyl); MS (EI) m/z: 450 (M+2\%), 381 (100), 210 (43), 193 (58), 165 (47), 147 (59), 120 (47), 93 (51), 91 (100), 81 (48); Anal. Calcd for $\mathrm{C}_{25} \mathrm{H}_{21} \mathrm{~F}_{3} \mathrm{~N}_{4} \mathrm{O}$ : C, 66.66; H, 4.70. Found: C, 66.32; H, 4.85 .

\section{6-Cyclopropylethynyl-1,9-di[(tert-butyldimethylsilyloxy)methyl]-1,3,6,9-tetrahydro-6-}

trifluoromethyl-2H-purin-2-one (4b). To a solution of 1,9-dihydro-1,9-di[(tertbutyldimethylsilyloxy)methyl]-6-trifluoromethyl-2H-purin-2-one $(114 \mathrm{mg}, 0.23 \mathrm{mmol})$ in dry THF ( $4 \mathrm{~mL}$ ) under $\mathrm{N}_{2}$ at $-78{ }^{\circ} \mathrm{C}$ was added cyclopropylethynylmagnesium bromide [generated in situ by adding cyclopropylethyne (140 mg, $1.27 \mathrm{mmol}, 60 \%$ solution in cyclohexane) in dry THF $(1 \mathrm{~mL})$ to a solution of ethylmagnesium bromide $(1.1 \mathrm{~mL}, 1.15 \mathrm{mmol}, 1.0 \mathrm{M}$ solution in THF) in dry THF ( $2 \mathrm{~mL})$ under $\mathrm{N}_{2}$ and refluxing the resulting mixture for 2 hours before cooling to ambient temperature]. After 30 minutes at $-78{ }^{\circ} \mathrm{C}$ and 30 minutes at ambient temperature, saturated aqueous ammonium chloride $(10 \mathrm{~mL})$ was added and the phases were separated. The aqueous phase was extracted with $\mathrm{CH}_{2} \mathrm{Cl}_{2}(3 \times 10 \mathrm{~mL})$ and the combined organic phases dried $\left(\mathrm{MgSO}_{4}\right)$ and evaporated in vacuo. Flash chromatography on silica gel eluting with EtOAc/hexane (1:2) gave the title compound (114 mg, 88\%) as colorless crystals; $166-9{ }^{\circ} \mathrm{C} ;{ }^{1} \mathrm{H}-$ NMR $\left(500 \mathrm{MHz} ; \mathrm{CDCl}_{3}\right) \delta 8.12(1 \mathrm{H}, \mathrm{s}, \mathrm{NH}), 7.20(1 \mathrm{H}, \mathrm{s}, 8-\mathrm{H}), 5.42\left[1 \mathrm{H}, \mathrm{d}, J\right.$ 9.6, $\mathrm{H}_{\mathrm{B}}$ in $\left.\mathrm{N}(1) \mathrm{CH}_{2}\right], 5.39\left[2 \mathrm{H}, \mathrm{s}, \mathrm{N}(9) \mathrm{CH}_{2}\right], 5.20\left[1 \mathrm{H}, \mathrm{d}, J\right.$ 9.6, $\mathrm{H}_{\mathrm{A}}$ in $\left.\mathrm{N}(1) \mathrm{CH}_{2}\right], 1.36(1 \mathrm{H}, \mathrm{m}, \mathrm{CH}$ in cyclopropyl), $0.83-0.38\left(22 \mathrm{H}, \mathrm{m}, 2 \times \mathrm{CH}_{2}\right.$ in cyclopropyl and $\mathrm{Me}$ in $\left.t-\mathrm{Bu}\right), 0.12(3 \mathrm{H}, \mathrm{s}, \mathrm{SiMe})$, 0.08 (3H, s, SiMe), 0.06 (3H, s, SiMe), 0.02 (3H, s, SiMe); ${ }^{19}$ F-NMR $\left(188 \mathrm{MHz} ; \mathrm{CDCl}_{3}\right) \delta-$ $80.12\left(\mathrm{~s}, \mathrm{CF}_{3}\right) ;{ }^{13} \mathrm{C}-\mathrm{NMR}\left(75 \mathrm{MHz} ; \mathrm{CDCl}_{3}\right) \delta 152.8$ (2-C), 131.9 (8-C), 127.8 (4-C), 123.7 (q, $J$ 287.6, $\mathrm{CF}_{3}$ ), 111.4 (5-C), 93.3 (三C-cyclopropyl), 70.0 [N(1) $\left.\mathrm{CH}_{2}\right], 68.6\left[\mathrm{~N}(9) \mathrm{CH}_{2}\right], 65.9$ (Pur$C \equiv), 61.9(\mathrm{q}, J 32.9,6-\mathrm{C}), 25.7(\mathrm{Me}$ in $t-\mathrm{Bu}), 25.3(\mathrm{Me}$ in $t-\mathrm{Bu}), 18.2(\mathrm{C}$ in $t-\mathrm{Bu}), 17.8(\mathrm{C}$ in $t-$ $\mathrm{Bu}), 8.5(2 \times \mathrm{CH} 2$ in cyclopropyl), -0.4 ( $\mathrm{CH}$ in cyclopropyl), $-5.2(\mathrm{SiMe}),-5.5(\mathrm{SiMe})$; MS (EI) m/z: $558\left(\mathrm{M}^{+}, 1 \%\right), 501$ (30), 225 (30), 75 (100), 73 (42); Anal. Calcd for $\mathrm{C}_{25} \mathrm{H}_{41} \mathrm{~F}_{3} \mathrm{~N}_{4} \mathrm{O}_{3} \mathrm{Si}_{2}$ : C, 53.73; H, 7.40. Found: C, 53.49; H, 7.54.

\section{6-Cyclopropylethynyl-1,9-di[(tert-butyldimethylsilyloxy)methyl]-8-phenyl-1,3,6,9-}

tetrahydro-6-trifluoromethyl-2H-purin-2-one (4c). To a solution of 1,9-dihydro-1,9-di[(tertbutyldimethylsilyloxy)methyl]-8-phenyl-6-trifluoromethyl-2H-purin-2-one (120 mg, $0.21 \mathrm{mmol}$ ) 
in dry THF ( $2 \mathrm{~mL}$ ) under $\mathrm{N}_{2}$ at $-78{ }^{\circ} \mathrm{C}$ was added cyclopropylethynyllithium [generated in situ by adding $n$-butyllithium $(0.54 \mathrm{~mL}, 0.84 \mathrm{mmol}, 1.6 \mathrm{M}$ solution in cyclohexane $)$ to cyclopropylethyne (90 mg, $1.05 \mathrm{mmol}, 80 \%$ solution in cyclohexane) in dry THF (2 mL) under $\mathrm{N}_{2}$ at $0{ }^{\circ} \mathrm{C}$ and stirring for $30 \mathrm{~min}$ at $0{ }^{\circ} \mathrm{C}$ and $1 \mathrm{~h}$ at ambient temperature]. After 30 minutes at $78{ }^{\circ} \mathrm{C}$ and 30 minutes at ambient temperature saturated aqueous ammonium chloride $(10 \mathrm{~mL})$ and $\mathrm{CH}_{2} \mathrm{Cl}_{2}(15 \mathrm{~mL})$ were added and the phases separated. The aqueous phase was extracted with $\mathrm{CH}_{2} \mathrm{Cl}_{2}(15 \mathrm{~mL})$ and the combined organic phases dried $\left(\mathrm{MgSO}_{4}\right)$ and evaporated in vacuo. Flash chromatography on silica gel eluting with EtOAc/hexane (1:4) gave the title compound (61 $\mathrm{mg}, 45 \%)$ as a colorless oil; $\mathrm{R}_{\mathrm{F}} 0.12(1: 5 \mathrm{EtOAc} / \mathrm{h}$ exane); $8.58(1 \mathrm{H}, \mathrm{s}, \mathrm{N}-\mathrm{H}), 7.6(2 \mathrm{H}, \mathrm{m}, \mathrm{CH}$ in $\mathrm{Ph}), 7.4-7.3(3 \mathrm{H}, \mathrm{m}, \mathrm{CH}$ in $\mathrm{Ph}), 5.45\left[1 \mathrm{H}, \mathrm{d}, J\right.$ 9.6, $\mathrm{H}_{\mathrm{B}}$ in $\left.\mathrm{N}(1) \mathrm{CH}_{2}\right], 5.43$ [2 H, s, N(9)CH $\mathrm{CH}_{2}$, 5.24 $\left[1 \mathrm{H}, \mathrm{d}, J\right.$ 9.6, HA in $\left.\mathrm{N}(1) \mathrm{CH}_{2}\right], 1.36(1 \mathrm{H}, \mathrm{m}, \mathrm{CH}$ in cyclopropyl), $0.88(9 \mathrm{H}, \mathrm{s}, \mathrm{Me}$ in $t-\mathrm{Bu}), 0.81$ $\left(13 \mathrm{H}, \mathrm{m}, 2 \times \mathrm{CH}_{2}\right.$ in cyclopropyl and $\mathrm{Me}$ in $\left.t-\mathrm{Bu}\right), 0.13(3 \mathrm{H}, \mathrm{s}, \mathrm{SiMe}), 0.090(3 \mathrm{H}, \mathrm{s}, \mathrm{SiMe}),-0.068$ $(3 \mathrm{H}, \mathrm{s}, \mathrm{SiMe}),-0.076(3 \mathrm{H}, \mathrm{s}, \mathrm{SiMe}) ;{ }^{19} \mathrm{~F}-\mathrm{NMR}\left(188 \mathrm{MHz} ; \mathrm{CDCl}_{3}\right) \delta-79.74\left(\mathrm{~s}, \mathrm{CF}_{3}\right) ;{ }^{13} \mathrm{C}-\mathrm{NMR}$ $\left(125 \mathrm{MHz} ; \mathrm{CDCl}_{3}\right) \delta 151.9$ (2-C), 143.5 (8-C), 129.5 (C in $\left.\mathrm{Ph}\right), 129.2(\mathrm{CH}$ in $\mathrm{Ph}), 129.1$ (4-C), 129.0 ( $\mathrm{CH}$ in $\mathrm{Ph}$ ), 128.6 (C in $\mathrm{Ph}$ ), 123.7 (q, $J$ 287.4, $\mathrm{CF}_{3}$ ), 111.3 (5-C), 93.3 (三C-cyclopropyl), $70.1\left[\mathrm{~N}(1) \mathrm{CH}_{2}\right], 68.4\left[\mathrm{~N}(9) \mathrm{CH}_{2}\right], 66.1$ (Pur-C $\left.\equiv\right)$ ), 62.0 (q, J 32.8, C-6), 25.8 (Me in $\left.t-\mathrm{Bu}\right), 25.4$ $(\mathrm{Me}$ in $t-\mathrm{Bu}), 18.3(\mathrm{C}$ in $t-\mathrm{Bu}), 17.8(\mathrm{C}$ in $t-\mathrm{Bu}), 8.5\left(2 \times \mathrm{CH}_{2}\right.$ in cyclopropyl), $-0.2(\mathrm{CH}$ in cyclopropyl), -5.1 (SiMe), -5.5 (SiMe), -5.6 (SiMe); MS (EI) m/z: 635 (M+1\%), 577 (34), 566 (44), 565 (98), 480 (33), 89 (51), 75 (45), 73 (100); HRMS: Found 634.3012, calc. for $\mathrm{C}_{31} \mathrm{H}_{45} \mathrm{~F}_{3} \mathrm{~N}_{4} \mathrm{O}_{3} \mathrm{Si}_{2} 634.2982$.

6-Cyclopropylethynyl-1,3,6,7-tetrahydro-6-trifluoromethyl-2H-purin-2-one (5a). To a solution of 6-cyclopropylethynyl-1,9-di[(tert-butyldimethylsilyloxy)methyl]-1,3,6,9-tetrahydro6-trifluoro-methyl-2H-purin-2-one in dry $\mathrm{MeCN}(4 \mathrm{~mL})$ was added tetramethylammonium fluoride $(1.1 \mathrm{~mL}, 0.5 \mathrm{M}$ solution in dry $\mathrm{MeCN})$. After 22 hours at ambient temperature the solvent was evaporated in vacuo and the product isolated by flash chromatography on silica gel eluting with $\mathrm{CHCl}_{3} / \mathrm{MeOH}(7: 1)$ to give the title compound $(27 \mathrm{mg}, 69 \%)$ as colorless crystals; $\mathrm{mp}>250{ }^{\circ} \mathrm{C}$ (decomp.); ${ }^{1} \mathrm{H}-\mathrm{NMR}(500 \mathrm{MHz}$; CD $\mathrm{OD}) \delta 7.42(1 \mathrm{H}, \mathrm{s}, 8-\mathrm{H}), 1.29(1 \mathrm{H}, \mathrm{m}, \mathrm{CH}$ in

cyclopropyl), $0.79\left(2 \mathrm{H}, \mathrm{m}, \mathrm{CH}_{2}\right.$ in cyclopropyl), $0.68\left(2 \mathrm{H}, \mathrm{m}, \mathrm{CH}_{2}\right.$ in cyclopropyl), ${ }^{19} \mathrm{~F}-\mathrm{NMR}$ $\left(188 \mathrm{MHz} ; \mathrm{CDCl}_{3}\right) \delta-80.34\left(\mathrm{~s}, \mathrm{CF}_{3}\right) ;{ }^{13} \mathrm{C}-\mathrm{NMR}\left(125 \mathrm{MHz} ; \mathrm{CD}_{3} \mathrm{OD} ; \mathrm{T}=50{ }^{\circ} \mathrm{C}\right) \delta 154.6(2-\mathrm{C})$, 138.7 (br, 4-C), 136.2 (8-C), 124.7 (q, J 284.4, $\mathrm{CF}_{3}$ ), 101.7 (br, 5-C), 92.7 (三C-cyclopropyl), 68.8 (Pur- $C \equiv), 57.6$ (q, J 34.9, 6-C), $8.6\left(2 \times \mathrm{CH}_{2}\right.$ in cyclopropyl), -0.2 ( $\mathrm{CH}$ in cyclopropyl); MS (EI) m/z: $270\left(\mathrm{M}^{+}, 7 \%\right), 202$ (12), 201 (100), 140 (90), 135 (10); Anal. Calcd for $\mathrm{C}_{11} \mathrm{H}_{9} \mathrm{~F}_{3} \mathrm{~N}_{4} \mathrm{O}$ : C, 48.89; H, 3.36. Found: C, 48.46; H, 3.53.

6-Cyclopropylethynyl-8-phenyl-1,3,6,7-tetrahydro-6-trifluoromethyl-2H-purin-2-one (5b). To a solution of 6-cyclopropylethynyl-1,9-di[(tert-butyldimethylsilyloxy)methyl]-8-phenyl1,3,6,9-tetrahydro-6-trifluoromethyl-2H-purin-2-one (47 mg, $0.074 \mathrm{mmol})$ in dry $\mathrm{MeCN}(2 \mathrm{~mL})$ was added tetramethylammonium fluoride $(0.6 \mathrm{~mL}, 0.5 \mathrm{M}$ solution in dry $\mathrm{MeCN}, 0.3 \mathrm{mmol})$. After 20 hours at ambient temperature, tetramethylammonium fluoride $(0.9 \mathrm{~mL}, 0.5 \mathrm{M}$ solution in dry $\mathrm{MeCN}, 0.45 \mathrm{mmol}$ ) was added, and after another 20 hours the solvent was evaporated in vacuo. Flash chromatography on silica gel eluting with $\mathrm{CHCl} / \mathrm{MeOH}$ (100:1) followed by 
$\mathrm{CHCl}_{3} / \mathrm{MeOH}(50: 1)$ and $\mathrm{CHCl}_{3} / \mathrm{MeOH}(25: 1)$ gave the title compound (18 $\left.\mathrm{mg}, 70 \%\right)$ as pale yellow crystals; mp $>150{ }^{\circ} \mathrm{C}$ (decomp.); RF 0.14 (25:1 $\left.\mathrm{CHCl}_{3} / \mathrm{MeOH}\right)$; ${ }^{1} \mathrm{H}-\mathrm{NMR}(300 \mathrm{MHz}$; $\left.\mathrm{CD}_{3} \mathrm{OD}\right) \delta$ 7.8-7.7 $(2 \mathrm{H}, \mathrm{m}, \mathrm{CH}$ in $\mathrm{Ph}), 7.4-7.2(3 \mathrm{H}, \mathrm{m}, \mathrm{CH}$ in $\mathrm{Ph}), 1.4-1.2(1 \mathrm{H}, \mathrm{m}, \mathrm{CH}$ in cyclopropyl), 0.9-0.6 (4H, m, $2 \times \mathrm{CH}_{2}$ in cyclopropyl); ${ }^{19} \mathrm{~F}-\mathrm{NMR}\left(188 \mathrm{MHz} ; \mathrm{CDCl}_{3}\right) \delta-81.58(\mathrm{~s}$, $\left.\mathrm{CF}_{3}\right) ;{ }^{13} \mathrm{C}-\mathrm{NMR}\left(125 \mathrm{MHz} ; \mathrm{CD}_{3} \mathrm{OD} ; \mathrm{T}=50{ }^{\circ} \mathrm{C}\right) \delta 154.5$ (2-C), 148.2 (8-C), 140.5 (br, 4-C), 131.1 (C in $\mathrm{Ph}), 130.1(\mathrm{CH}$ in $\mathrm{Ph}), 129.8(\mathrm{CH}$ in $\mathrm{Ph}), 126.9(\mathrm{CH}$ in $\mathrm{Ph}), 124.7\left(\mathrm{q}, J\right.$ 284.9, $\left.\mathrm{CF}_{3}\right)$, 101.5 (br, 5-C), 92.8 (三C-cyclopropyl), 68.9 (Pur- $C \equiv), 57.6$ (br, 6-C), 8.7 ( $2 \times \mathrm{CH}_{2}$ in cyclopropyl), -0.2 (CH in cyclopropyl); MS (EI) m/z: $346\left(\mathrm{M}^{+}, 3 \%\right), 278$ (17), 277 (100), 171 (14), 104 (10), 77 (8); HRMS: Found 346.1027, calc. for $\mathrm{C}_{17} \mathrm{H}_{13} \mathrm{~F}_{3} \mathrm{~N}_{4} \mathrm{O} 346.1041$.

6-Cyclopropylethynyl-1,9-dibenzyl-1,3,6,9-tetrahydro-2H-purin-2-one (6a). To a solution of 1,9-dibenzyl-1,9-dihydro-2 $\mathrm{H}$-purin-2-one $(158 \mathrm{mg}, 0.5 \mathrm{mmol})$ in dry THF $(8 \mathrm{~mL})$ under $\mathrm{N}_{2}$ at $78{ }^{\circ} \mathrm{C}$ was added cyclopropylethynylmagnesium bromide [generated in situ by adding cyclopropylethyne (220 mg, $2.0 \mathrm{mmol}, 60 \%$ solution in cyclohexane) in dry THF (1 mL) to a solution of ethylmagnesium bromide $(2.0 \mathrm{~mL}, 2.0 \mathrm{mmol}, 1.0 \mathrm{M}$ solution in THF) in dry THF (2 $\mathrm{mL}$ ) under $\mathrm{N}_{2}$ and refluxing the resulting mixture for 2 hours before cooling to ambient temperature]. After stirring for 30 minutes at $-78{ }^{\circ} \mathrm{C}$ and 30 minutes at ambient temperature, saturated aqueous ammonium chloride $(10 \mathrm{~mL})$ was added to the reaction mixture and the phases were separated. The aqueous phase was extracted with $\mathrm{CH}_{2} \mathrm{Cl}_{2}(3 \times 10 \mathrm{~mL})$ and the combined organic phases were dried $\left(\mathrm{MgSO}_{4}\right)$ and evaporated in vacuo. Flash chromatography on silica gel eluting with EtOAc/hexane (3:1) gave the title compound $(157 \mathrm{mg}, 82 \%)$ as yellow crystals; $\mathrm{mp}$ 120-2 ${ }^{\circ} \mathrm{C}$; RF 0.18 (3:1 EtOAc/hexane); ${ }^{1} \mathrm{H}-\mathrm{NMR}\left(300 \mathrm{MHz} ; \mathrm{CDCl}_{3}\right) \delta 9.78(1 \mathrm{H}, \mathrm{s}, \mathrm{NH}) 7.4-7.2$ $(10 \mathrm{H}, \mathrm{m}, \mathrm{CH}$ in $\mathrm{Ph}), 7.10(1 \mathrm{H}, \mathrm{s}, 8-\mathrm{H}), 5.29(1 \mathrm{H}, \mathrm{d}, J 1.5,6-\mathrm{H}), 5.25[1 \mathrm{H}, \mathrm{d}, J 15.1, \mathrm{HB}$ in $\left.\mathrm{N}(1) \mathrm{CH}_{2}\right], 5.00\left[2 \mathrm{H}, \mathrm{s}, \mathrm{N}(9) \mathrm{CH}_{2}\right], 4.27\left[1 \mathrm{H}, \mathrm{d}, J 15.1, \mathrm{H}_{\mathrm{A}}\right.$ in $\left.\mathrm{N}(1) \mathrm{CH}_{2}\right], 1.24(1 \mathrm{H}, \mathrm{m}, \mathrm{CH}$ in cyclopropyl), $0.73\left(2 \mathrm{H}, \mathrm{m}, \mathrm{CH}_{2}\right.$ in cyclopropyl), $0.66\left(2 \mathrm{H}, \mathrm{m}, \mathrm{CH}_{2}\right.$ in cyclopropyl); ${ }^{13} \mathrm{CNMR}(75$ $\left.\mathrm{MHz} ; \mathrm{CDCl}_{3}\right) \delta 153.8(2-\mathrm{C}), 136.5(\mathrm{C}$ in $\mathrm{Ph}), 135.3(\mathrm{C}$ in $\mathrm{Ph}), 131.9(8-\mathrm{C}), 128.8(\mathrm{CH}$ in $\mathrm{Ph})$, $128.4(\mathrm{CH}$ in $\mathrm{Ph}), 128.1(\mathrm{CH}$ in $\mathrm{Ph}), 128.0(\mathrm{CH}$ in $\mathrm{Ph}), 127.5(\mathrm{CH}$ in $\mathrm{Ph}), 127.4(\mathrm{CH}$ in $\mathrm{Ph})$, 126.4 (4-C), 112.9 (5-C), 89.0 (三C-cyclopropyl), 71.8 (Pur- $C \equiv), 48.9$ (6-C), 48.3 [N(1) $\mathrm{CH}_{2}$ ], $47.5\left[\mathrm{~N}(9) \mathrm{CH}_{2}\right], 8.3\left(\mathrm{CH}_{2}\right.$ in cyclopropyl), $8.2\left(\mathrm{CH}_{2}\right.$ in cyclopropyl), $-0.5(\mathrm{CH}$ in cyclopropyl); MS (EI) m/z: 382 (M+, 26\%), 381 (11), 291 (43), 249 (11), 225 (14), 221 (13), 140 (69), 91 (100); HRMS: Found 382.1791, calc. for $\mathrm{C}_{24} \mathrm{H}_{22} \mathrm{~N}_{4} \mathrm{O} 382.1794$.

6-Cyclopropylethynyl-1,9-dibenzyl-1,9-dihydro-2H-purin-2-one (7a). To a solution of 6cyclopropylethynyl-1,9-dibenzyl-1,3,6,9-tetrahydro-2H-purin-2-one (60 mg, $0.16 \mathrm{mmol})$ in dry $\mathrm{CH}_{2} \mathrm{Cl}_{2}(5 \mathrm{~mL})$ was added $\mathrm{MnO}_{2}(600 \mathrm{mg}, 6.9 \mathrm{mmol})$. The resulting mixture was stirred at ambient temperature for 23 hours, filtered and the product was isolated by flash chromatography on silica gel eluting with $\mathrm{CHCl}_{3} / \mathrm{MeCN}$ (3:1) to give the title compound (40 $\mathrm{mg}, 67 \%$ ) as yellow crystals; mp $136-7{ }^{\circ} \mathrm{C} ;{ }^{1} \mathrm{H}-\mathrm{NMR}\left(300 \mathrm{MHz} ; \mathrm{CDCl}_{3}\right) \delta 7.71(1 \mathrm{H}, \mathrm{s}, 8-\mathrm{H}), 7.2-7.4(10 \mathrm{H}, \mathrm{m}, \mathrm{CH}$ in $\mathrm{Ph}), 5.45\left[2 \mathrm{H}, \mathrm{s}, \mathrm{N}(1) \mathrm{CH}_{2}\right], 5.11\left[2 \mathrm{H}, \mathrm{s}, \mathrm{N}(9) \mathrm{CH}_{2}\right], 1.57$ (1H, m, CH in cyclopropyl), 1.00 (2H, $\mathrm{m}, \mathrm{CH}_{2}$ in cyclopropyl), $0.88\left(2 \mathrm{H}, \mathrm{m}, \mathrm{CH}_{2}\right.$ in cyclopropyl); ${ }^{13} \mathrm{C}-\mathrm{NMR}\left(75 \mathrm{MHz} ; \mathrm{CDCl}_{3}\right) \delta 157.6$ (4-C), 156.1 (2-C), 146.4 (8C), 136.0 (C in $\mathrm{Ph}), 134.7$ (C in $\mathrm{Ph}), 134.4$ (6-C), 129.0 (CH in $\mathrm{Ph}$ ), $128.4(2 \times \mathrm{CH}$ in $\mathrm{Ph}), 128.0(\mathrm{CH}$ in $\mathrm{Ph}), 127.7(\mathrm{CH}$ in $\mathrm{Ph}), 127.6(\mathrm{CH}$ in $\mathrm{Ph}), 126.1(5-\mathrm{C}), 115.9$ 
(三C-cyclopropyl), 65.9 (Pur- $C \equiv), 51.6\left[\mathrm{~N}(1) \mathrm{CH}_{2}\right], 46.5\left[\mathrm{~N}(9) \mathrm{CH}_{2}\right], 10.1\left(2 \times \mathrm{CH}_{2}\right.$ in cyclopropyl), 0.9 (CH in cyclopropyl); MS (EI) m/z: 380 (M+, 45\%), 379 (22), 289 (35), 155 (18), 91 (100), 65 (19); Anal. Calcd for $\mathrm{C}_{24} \mathrm{H}_{20} \mathrm{~N}_{4} \mathrm{O}$ : C, 75.77; H, 5.30. Found: C, 75.59; H, 5.40.

6-Cyclopropylethynyl-1,9-dihydro-1,9-di[(tert-butyldimethylsilyloxy)methyl]-2H-purin-2one (7b). To a solution of 1,9-dihydro-1,9-di[(tert-butyldimethylsilyloxy)methyl]-2H-purin-2one (100 mg, $0.32 \mathrm{mmol})$ in dry THF $(4 \mathrm{~mL})$ under $\mathrm{N}_{2}$ at $-78{ }^{\circ} \mathrm{C}$ was added dropwise a solution of cyclopropylethynylmagnesium chloride $(0.48 \mathrm{mmol})$ in dry THF $(4 \mathrm{~mL})$ [generated in situ by adding cyclopropylethyne ( $56 \mathrm{mg}, 0.51 \mathrm{mmol}, 60 \%$ solution in cyclohexane) in dry THF ( $2 \mathrm{~mL}$ ) to a solution of isopropylmagnesium chloride $(0.24 \mathrm{~mL}, 0.48 \mathrm{mmol}, 2.0 \mathrm{M}$ solution in THF) in dry THF $(2 \mathrm{~mL})$ under $\mathrm{N}_{2}$ and refluxing the resulting mixture for 2 hours before cooling to ambient temperature]. After stirring for 1 hour at $-78{ }^{\circ} \mathrm{C}$ and 20 hours at ambient temperature, saturated aqueous ammonium chloride $(10 \mathrm{~mL})$ and $\mathrm{CH}_{2} \mathrm{Cl}_{2}(20 \mathrm{~mL})$ were added to the reaction mixture and the phases were separated. The organic phase was extracted with saturated sodium hydrogen carbonate $(10 \mathrm{~mL})$, dried $\left(\mathrm{MgSO}_{4}\right)$ and evaporated in vacuo. The crude product $(6 \mathrm{~b})$ was dissolved in dry benzene $(20 \mathrm{~mL})$. DDQ $(72 \mathrm{mg}, 0.32 \mathrm{mmol})$ was added and the resulting mixture was stirred at ambient temperature for 3 hours, filtered and the solvent was evaporated in vacuo. The product was isolated by flash chromatography on silica gel eluting with EtOAc/hexane $(2: 1)$ to give the title compound $(73 \mathrm{mg}, 47 \%)$ as orange crystals; $\mathrm{mp} 140-2{ }^{\circ} \mathrm{C}$; ${ }^{1} \mathrm{H}-\mathrm{NMR}\left(500 \mathrm{MHz} ; \mathrm{CDCl}_{3}\right) \delta 8.10(1 \mathrm{H}, \mathrm{s}, 8-\mathrm{H}), 5.76$ [2H, s, N(1)CH $\mathrm{CH}_{2}, 5.51$ [2H, s, N(9) $\mathrm{CH}_{2}$ ], $1.66\left(1 \mathrm{H}, \mathrm{m}, \mathrm{CH}\right.$ in cyclopropyl), $1.04\left(4 \mathrm{H}, \mathrm{m}, 2 \times \mathrm{CH}_{2}\right.$ in cyclopropyl), $0.86(9 \mathrm{H}, \mathrm{s}, \mathrm{Me}$ in $t-\mathrm{Bu})$, $0.85(9 \mathrm{H}, \mathrm{s}, \mathrm{Me}$ in $t-\mathrm{Bu}), 0.12(6 \mathrm{H}, \mathrm{s}, 2 \times \mathrm{SiMe}), 0.08(6 \mathrm{H}, \mathrm{s}, 2 \times \mathrm{SiMe}) ;{ }^{13} \mathrm{C}-\mathrm{NMR}(75 \mathrm{MHz}$; $\left.\mathrm{CDCl}_{3}\right) \delta 157.9$ (4-C), 155.3 (2-C), 146.2 (8C), 134.0 (6-C), 126.1 (5-C), 114.6 (三Ccyclopropyl), $71.7\left[\mathrm{~N}(1) \mathrm{CH}_{2}\right], 66.8\left[\mathrm{~N}(9) \mathrm{CH}_{2}\right], 65.4$ (Pur- $\left.C \equiv\right), 25.6$ (Me in $\left.t-\mathrm{Bu}\right), 25.5$ (Me in $t-$ $\mathrm{Bu}), 18.0(\mathrm{C}$ in $t-\mathrm{Bu}), 17.9(\mathrm{C}$ in $t-\mathrm{Bu}), 10.1\left(2 \times \mathrm{CH}_{2}\right.$ in cyclopropyl), $1.0(\mathrm{CH}$ in cyclopropyl), 5.3 (4×SiMe); MS (EI) m/z: 488 (M+1\%), 432 (34), 431 (100), 401 (79), 135 (79), 73 (37); Anal. Calcd for $\mathrm{C}_{24} \mathrm{H}_{40} \mathrm{~N}_{4} \mathrm{O}_{3} \mathrm{Si}_{2}$ : C, 58.97; H, 8.25. Found: C, 58.70; H, 8.35.

\section{Acknowledgements}

The purchase of the 200, 300 and $500 \mathrm{MHz}$ Bruker Spectrospin Avance instruments used in this study, was made possible through financial support from The Research Council of Norway (NFR).

\section{References}

1. For recent reviews on NNRTIs, see for instance: (a) Högberg, M.; Morrison, I. Exp. Opin. Ther. Pat. 2000, 10, 1189. (b) Pedersen, O. S.; Pedersen, E. B. Synthesis 2000, 479. (c) De 
Clerq, E. Antiviral Res. 1998, 38, 152. (d) Tucker, T. J.; Lumma, W. C.; Culbertson, J. C. Methods Enzymol. 1996, 275, 440. (e) Pedersen, O. S.; Pedersen, E. B. Antiviral Chem. Chemother. 1999, 10, 285.

2. Tucker, T. J.; Lyle, T. A.; Wiscount, C. M.; Britcher, S. F.; Young, S-D.; Sanders, W. M.; Lumma, W. C.; Goldman, M. E.; O'Brien, J. A.; Ball, R. G.; Homnick, C. F.; Schleif, W. A.; Emini, E. A.; Huff, J. R.; Anderson, P. S. J. Med. Chem. 1994, 37, 2437.

3. (a) Corbett, J. W.; Ko, S. S.; Rodgers, J. D.; Jeffrey, S.; Bacheler, L. T.; Klabe, R. M.; Diamond, S.; Lai, C.-M.; Rabel, S. R.; Saye, J. A.; Adams, S. P.; Trainor, G. L.; Anderson, P. S.; Erickson-Viitanen, S. K. Antimicrob. Agents Chemother. 1999, 43, 2893. (b) Corbett, J. W.; Ko, S. S.; Rodgers, J. D.; Gearhart, L. A.; Magnus, N. A.; Bacheler, L. T.; Diamond, S.; Jeffrey, S.; Klabe, R. M.; Cordova, B. C.; Garber, S.; Logue, K.; Trainor, G. L.; Anderson, P. S.; Erickson-Viitanen, S. K. E. J. Med. Chem. 2000, 43, 2019. (c) Magnus, N. A.; Confalone, P. N.; Storace, L. Tetrahedron Lett. 2000, 41, 3015. (d) Kauffman, G. S.; Harris, G. D.; Dorow, R. L.; Stone, B. R. P.; Parsons, R. L.; Pesti, J. A.; Magnus, N. A.; Fortunak, J. M.; Confalone, P. N.; Nugent, W. A. Org. Lett. 2000, 2, 3119.

4. Andresen, G.; Gundersen, L.-L.; Rise, F. Tetrahedron 1996, 52, 12979.

5. Andresen, G.; Dalhus, B.; Eriksen, A. B. Gundersen, L.-L.; Rise, F. J. Chem. Soc., Perkin Trans. 1. In press.

6. (a) Prakash, G. K. S.; Krishnamurti, R.; Olah, G. A. J. Am. Chem. Soc. 1989, 111, 393. (b) Krishnamurti, R.; Bellew, D. R.; Prakash, G. K. S. J. Org. Chem. 1991, 54, 984. (c) Kotun, S. P.; Anderson, J. D. O.; DesMarteau, D. D. J. Org. Chem. 1992, 57, 1124.

7. Stahly, G. P.; Bell, D. R. J. Org. Chem. 1989, 54, 2873.

8. Corley, E. G.; Thomson, A. S.; Huntington, M. Org. Synth. 2000, 77, 231.

9. For other examples of Suzuki couplings on halopurines see : (a) Havelkova, M., Hocek, M.; Cesnek, M.; Dvorak, D. Synlett 1999, 7, 1145. (b) Hocek, M.; Holy, A.; Votruba, I.; Dvorakova, H. J. Med. Chem. 2000, 43, 1817. (c) Cesnek, M.; Hocek, M.; Holy, A. Coll. Czech. Chem. Commun. 2000, 65, 1357.

10. For other examples of Pd-catalyzed coupling between halopurines and other organometallic reagents, see: (a) Langli, G.; Gundersen, L.-L.; Rise, F. Tetrahedron, 1996, 52, 5625, and references therein. (b) Nolsøe, J. M. J.; Gundersen, L.-L.; Rise, F. Acta Chem. Scand. 1999, 53, 366. 